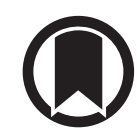

CrossMark

\title{
Treatment of latent tuberculosis with 12 weeks isoniazid/rifapentine in clinical practice
}

\author{
To the Editor:
}

As part of the global End TB strategy [1-3], treatment of latent tuberculosis infection (LTBI) is now recommended in low endemic middle- and high-income countries for certain risk groups, such as contacts of pulmonary TB, migrants from high endemic countries and immunocompromised individuals.

The US Food and Drug Administration approved the rifamycin rifapentine (Priftin; Sanofi, Paris, France) (P) for treatment of active tuberculosis (TB) in 1998 and for LTBI in 2014 (www.fda.gov). P is as effective as rifampicin (R) but with a five times longer half-life. Therefore, $\mathrm{P}$ is administered intermittently and should be taken together with a fat-containing meal for optimal absorption. In a recent meta-analysis, $\mathrm{P}$ (20-30 mg.kg ${ }^{-1}$, maximum $900 \mathrm{mg}$ ) and high-dose isoniazid (H) (15 mg.kg-1, maximum $900 \mathrm{mg}$ ) once a week for 12 weeks (3HP) provided by direct observed therapy (DOT) was as effective as other recommended treatments for LTBI, i.e. 9 months $\mathrm{H}(9 \mathrm{H}), 4$ months $\mathrm{R}(4 \mathrm{R})$ and 3 months $\mathrm{H}$ and $\mathrm{R}(3 \mathrm{HR})$ (OR 0.89 ) and associated with higher completion rate (87.5\% versus $65.9 \%$ ) [4]. Overall, $3 \mathrm{HP}$ was associated with lowest frequency of reported adverse events (AEs) (11.5\%), including hepatotoxicity (1\%). However, higher frequency of flu-like symptoms have been reported, as well as severe AEs (6\%) even though comparisons were limited by variability in definitions and inconsistent data [5].

In 2015, 3HP was introduced as an alternative regimen to standard treatment with $9 \mathrm{H}$, $4 \mathrm{R}$ or $3 \mathrm{HR}$ for LTBI at the TB Center, Karolinska University Hospital in Stockholm, Sweden. 3HP is at present not recommended for pregnant or breastfeeding women, or in patients with severe immunodeficiency. However, studies on $3 \mathrm{HP}$ versus $9 \mathrm{H}$ among moderately immunocompromised HIV-infected persons have shown that $3 \mathrm{HP}$ is more effective ( 0.39 versus 1.25 active TB cases per person-year) and also associated with higher completion rates (89\% versus 64\%) [6]. Data on pregnant women inadvertently exposed to $3 \mathrm{HP}$ have not shown higher frequency of fetal loss or anomalies [7]. Further studies are ongoing in more severely immunocompromised HIV-infected persons and pregnant/post partum women (www.clinicaltrials. gov). There is also recent data available on non-DOT administrations. In a study from the USA, completion rate was noninferior for self-administration as compared to DOT (77.9\% versus 85.4\%) [8].

$\mathrm{P}$ is not yet approved by the European Medicines Agency (EMA) but has been approved by the Swedish Medical Products Agency as a licensed drug since 2013. Our objective was to evaluate feasibility, adherence and AEs with $3 \mathrm{HP}$ treatment of LTBI in clinical practice.

This was a retrospective, observational study of $3 \mathrm{HP}$ as treatment of LTBI in clinical practice. HIV-negative migrants from high endemic countries and pulmonary TB contacts with a positive tuberculin skin test or QuantiFERON In-Tube Gold (Qiagen, Hilden, Germany) test were offered 3HP as an alternative to standard regiments for treatment of LTBI according to current clinical recommendations [9]. 3HP (Priftin (Sanofi) and Tibinide (Meda, Solna, Sweden)) was given as DOT on one specific day of the week for 12 consecutive weeks. Patients were provided HP by the responsible TB nurse and treatment was given under supervision together with a fat-containing nutritional drink or sandwich. Contraindications to $3 \mathrm{HP}$ were suspected active TB, exposure to MDR-TB, abnormal liver function tests, previous AEs to $\mathrm{H}$ or $\mathrm{R}$, pregnancy/breastfeeding, HIV or other immunodeficiency, on-going drug abuse, or unwillingness to comply with DOT. Liver function test results, AEs, adherence to DOT and exceptions

@ERSpublications

Completion rate with 12 weeks isoniazid/rifapentine for latent tuberculosis was high. Direct observed therapy was solved with flexibility. Adverse events were mostly mild, except one case of suspected nephrotoxicity, which calls for heightened attention. http://ow.ly/DRE430mw5QQ

Cite this article as: Fröberg G, Jansson L, Bruchfeld J. Treatment of latent tuberculosis with 12 weeks isoniazid/rifapentine in clinical practice. Eur Respir $J$ 2019; 53: 1801128 [https://doi.org/10.1183/ 13993003.01128-2018]. 
from regular administration (e.g. shortage of drugs) were documented on separate patient report forms every administration day and in the patient's regular file when needed.

AEs were graded 0-4 according to an in-house system based on severity and duration of symptoms (0: none; 1 : mild, <1 month; 2: mild, >1 month; 3: severe, pause in treatment needed; 4: severe, discontinued treatment). The study was approved by Stockholm Ethical Review Board (2018/349-31).

Between May 2015 and December 2017, a total of 30 patients initiated treatment with 3HP. During the same period about 650 patients in total initiated LTBI treatment, out of which 170 were migrants from high endemic countries and pulmonary TB contacts. As such, about 5\% (30 out of 650 ) of all patients and about $18 \%$ (30 out of 170) of young migrants and contacts that initiated LTBI treatment were given $3 \mathrm{HP}$, while the remaining were given standard treatment with $9 \mathrm{H}, 4 \mathrm{R}$ or $3 \mathrm{HR}$.

Clinical characteristics and AEs for patients initiating 3HP are presented in table 1. Most were young adults $<25$ years old (median 23.8 years, range $17-44$ years) and the majority were males. The majority were migrants from high TB endemic countries, such as Somalia, Eritrea, Afghanistan and the Democratic Republic of Congo. 27 (90\%) out of 30 patients completed treatment. 21 (70\%) out of 30 patients experienced any AEs.

The most common AEs were nausea/stomach ache $(n=13)$, headache/vertigo $(n=10)$, rash/itching $(n=3)$, flu-like symptoms $(\mathrm{n}=3)$ and slightly elevated liver enzymes (alanine transaminase maximum $94.8 \mathrm{IU} \cdot \mathrm{L}^{-1}$ ) $(n=2)$. Three $(10 \%)$ out of 30 discontinued due to more severe AEs. One 32-year-old woman experienced severe nausea. One 44-year-old woman developed urticaria but no other anaphylactic symptoms. One 20 -year-old man developed fever, nausea, stomach pain and elevated creatinine $\left(180 \mu \mathrm{mol} \cdot \mathrm{L}^{-1}\right) 6 \mathrm{~h}$ after the first dose of $3 \mathrm{HP}$ and progressive kidney failure necessitated temporary dialysis with full recovery after 3 months. In the diagnostic work-up, it was noted that he had been admitted to the emergency department due to stomach pain and elevated creatinine $\left(106 \mu \mathrm{mol} \cdot \mathrm{L}^{-1}\right)$ after a single dose of $\mathrm{R} 1 \mathrm{month}$ prior to 3HP but with full recovery within a week. This event was unfortunately overlooked prior to initiation of HP. R-associated nephrotoxicity has been described previously as a rare adverse event $(<1$ per 1000) $[10,11]$. With re-exposure, anti- $R$ antibodies activate the complement cascade with target-cell damage. This has, to our knowledge, not been described for $\mathrm{P}$ alone before. However, a rifamycin immunological cross-reactivity is likely, with potential deterioration of a previous R-induced nephritis.

A challenge to adherence was the necessity of DOT and long travel distances to our unit. This was solved by providing the last doses by telephone-DOT or primary healthcare ( $\mathrm{PHC}$ ) for two patients or public transport tickets to our clinic for three patients. Shortage of $\mathrm{P}$ during a limited period was another obstacle and therefore clinic routine is now to set aside a complete 12-week treatment for each patient before initiation.

TABLE 1 Characteristics and adverse events (AEs) of patients treated with 3 months of isoniazid $(\mathrm{H})$ and rifapentine $(\mathrm{P})$

Patients treated with HP n

Patients that reported AEs $\mathrm{n}$

\begin{tabular}{|c|c|c|}
\hline Total & 30 & 21 \\
\hline \multicolumn{3}{|l|}{ Sex } \\
\hline Male & 20 & 12 \\
\hline Female & 10 & 9 \\
\hline \multicolumn{3}{|l|}{ Origin } \\
\hline Europe & 3 & 2 \\
\hline Africa & 20 & 16 \\
\hline Asia/Southeast Asia & 7 & 3 \\
\hline \multicolumn{3}{|l|}{ Treatment group } \\
\hline Migrants & 25 & 16 \\
\hline Contacts & 5 & 5 \\
\hline \multicolumn{3}{|l|}{ AE grade } \\
\hline Grade 1 & & 9 \\
\hline Grade 2 & & 8 \\
\hline Grade 3 & & 1 \\
\hline Grade 4 & & 3 \\
\hline
\end{tabular}

AE grades were as follows. 0: none; 1: mild, <1 month; 2 : mild, $>1$ month; 3 : severe, pause in treatment needed; 4: severe, discontinued treatment. 
Completion rate with $3 \mathrm{HP}$ was high $(90 \%)$ in this group with mostly young migrants from Africa, where discontinuation with standard LTBI treatment has been a challenge at Karolinska [12]. However, the study group was small and consisted mainly of younger patients, which limits the generalisability of our findings to other age groups. In addition, no control group receiving standard regimens was included and protective efficacy was not possible to evaluate. AEs were mostly mild and temporary. However, one patient developed acute kidney failure, probably induced by a previous rare AE to R, which calls for heightened attention. DOT can be challenging and alternative solutions via telephone DOT or PHC can facilitate treatment. Despite one event with nephrotoxicity, we consider $3 \mathrm{HP}$ as a promising alternative to longer LTBI treatments, especially in young migrants, and as such, an improved tool in the global effort to eliminate TB. We advocate approval of $\mathrm{P}$ by the EMA to facilitate introduction of $3 \mathrm{HP}$ in Europe and also suggest a centralised European report system to monitor rare AEs.

Gabrielle Fröberg $\odot^{1,2}$, Lena Jansson ${ }^{1}$ and Judith Bruchfeld ${ }^{1,2}$

${ }^{1}$ Dept of Infectious Diseases, Karolinska University Hospital, Stockholm, Sweden. ${ }^{2}$ Division of Infectious Diseases, Dept of Medicine Solna, Karolinska Institutet, Stockholm, Sweden.

Correspondence: Gabrielle Fröberg, Dept of Infectious Diseases, B2:00, Karolinska University Hospital, 17176 Stockholm, Sweden. E-mail: gabrielle.froberg@sll.se

Received: June 152018 | Accepted after revision: Oct 242018

Conflict of interest: None declared.

\section{References}

WHO. Implementing the End TB Strategy: the Essentials. Geneva, World Health Organization, 2015.

2 Getahun H, Matteelli A, Abubakar I, et al. Management of latent Mycobacterium tuberculosis infection: WHO guidelines for low tuberculosis burden countries. Eur Respir J 2015; 46: 1563-1576.

3 Zenner D, Loutet MG, Harris R, et al. Evaluating 17 years of latent tuberculosis infection screening in north-west England: a retrospective cohort study of reactivation. Eur Respir J 2017; 50.

4 Njie GJ, Morris SB, Woodruff RY, et al. Isoniazid-rifapentine for latent tuberculosis infection: a systematic review and meta-analysis. Am J Prev Med 2018; 55: 244-252.

5 Pease C, Hutton B, Yazdi F, et al. A systematic review of adverse events of rifapentine and isoniazid compared to other treatments for latent tuberculosis infection. Pharmacoepidemiol Drug Saf 2018; 27: 557-566.

6 Sterling TR, Scott NA, Miro JM, et al. Three months of weekly rifapentine and isoniazid for treatment of Mycobacterium tuberculosis infection in HIV-coinfected persons. AIDS 2016; 30: 1607-1615.

7 Moro RN, Scott NA, Vernon A, et al. Exposure to latent tuberculosis treatment during pregnancy. The PREVENT TB and the iAdhere Trials. Ann Am Thorac Soc 2018; 15: 570-580.

8 Belknap R, Holland D, Feng PJ, et al. Self-administered versus directly observed once-weekly isoniazid and rifapentine treatment of latent tuberculosis infection: a randomized trial. Ann Intern Med 2017; 167: 689-697.

9 Folkhälsomyndigheten. Rekommendationer för preventiva insatser mot tuberkulos - hälsokontroll, smittspårning, behandling av latent infektion och vaccination [Recommendations for preventative action against tuberculosis health checks, infection tracking, treatment of latent infection and vaccination]. Solna, Folkhälsomyndigheten, 2017.

10 Manika K, Tasiopoulou K, Vlogiaris L, et al. Rifampicin-associated acute renal failure and hemolysis: a rather uncommon but severe complication. Ren Fail 2013; 35: 1179-1181.

11 Chiba S, Tsuchiya K, Sakashita H, et al. Rifampicin-induced acute kidney injury during the initial treatment for pulmonary tuberculosis: a case report and literature review. Intern Med 2013; 52: 2457-2460.

12 Kan B, Kalin M, Bruchfeld J. Completing treatment for latent tuberculosis: patient background matters. Int $J$ Tuberc Lung Dis 2013; 17: 597-602. 